\title{
Laboratório Remoto de Robótica como Elemento Motivador para a Aprendizagem de Programação
}

\author{
Thais Oliveira Almeida ${ }^{1,2}$, José Francisco de Magalhães Netto ${ }^{2}$, Romero Gomes da \\ Silva $^{3}$, Tiago de Paula Custódio ${ }^{2}$ \\ ${ }^{1}$ Departamento de Ciência da Computação - Universidade Federal de Roraima (UFRR) \\ - 69.310-000 - Boa Vista - RR - Brasil \\ ${ }^{2}$ Programa de Pós Graduação em Informática (PPGI) - Universidade Federal do \\ Amazonas (UFAM) - 69.067-005 - Manaus - AM - Brasil. \\ ${ }^{3}$ Instituto Federal de Educação, Ciência e Tecnologia de Roraima (IFRR) - 69.303-340 \\ - Boa Vista - RR - Brasil. \\ thais.oliveira@ufrr.br, jnettodicomp.ufam.edu.br, \\ romero.ufrregmail.com, tpceicomp.ufam.edu.br
}

\begin{abstract}
This paper presents the development of a remote robotics laboratory that supports students in programming learning. The laboratory is available in a Learning Management System (LMS) accessed via Web allowing many people to have experience with experiments of this nature. The system explores Educational Robotics (ER) as a motivating element of learning. Its features were tested with promising results, where users felt motivated to continue learning programming and robotics.
\end{abstract}

Resumo. Este artigo apresenta o desenvolvimento de um laboratório remoto de robótica que apoia alunos na aprendizagem de programação. $O$ laboratório encontra-se disponivel em um Ambiente Virtual de Aprendizagem (AVA) acessado via Web permitindo que várias pessoas tenham experiência com experimentos desta natureza. O sistema explora a Robótica Educacional (RE) como elemento motivador de aprendizagem. Suas funcionalidades foram testadas com resultados promissores, onde os alunos sentiram-se motivados a continuar aprendendo programação e robótica.

\section{Introdução}

De acordo com o Computer Science Teachers Association ${ }^{1}$ [CSTA 2013] a temática mais abordada nos diversos cursos de informática é a programação com $81 \%$. Este fato revela a importância que a programação tem dentro do campo da informática. Antes dos alunos iniciarem a programar, eles precisam desenvolver o raciocínio lógico por meio de algoritmos. Para ser bem sucedido em cursos introdutórios de programação, o aluno deve aprender um conjunto de conceitos básicos, a sintaxe de uma linguagem de programação e desenvolver habilidades para solucionar problemas [Santos et al. 2013].

\footnotetext{
${ }^{1}$ http://www.csteachers.org/
} 
Após tentativas frustradas de desenvolver programas para resolver problemas, o aluno reinicia suas ações em busca de uma solução correta, tentando descobrir e corrigir os erros cometidos, inicialmente, em um processo de tentativa e erro, até que consiga ganhar experiência nesse processo que lhe permita tomar consciência sobre o mesmo e sobre a construção de soluções corretas [Tavares et al. 2012]. Em uma tentativa de amenizar estas dificuldades, torna-se necessário pensar em novas estratégias para o ensino da programação, centradas nos interesses dos alunos.

Em computação a robótica engloba diferentes tópicos que podem ser abordados para o desenvolvimento de um robô, com foco no software que este robô irá possuir que determina em um nível mais alto na hierarquia em relação ao hardware - como o robô irá funcionar [Azevedo et al. 2010]. Segundo [D'Abreu et al. 2011] o desenvolvimento de atividades desta natureza envolve pelo menos a concepção, implementação, construção, automação e controle do dispositivo desenvolvido.

Neste sentido, a Robótica Educacional (RE) vem sendo utilizada como técnica de aprendizado que permite desenvolver atividades, e como ferramenta que estimula a criatividade dos alunos devido a sua natureza dinâmica, interativa e até mesmo lúdica, além de servir de motivador para estimular o interesse dos alunos no ensino tradicional [Gomes et al. 2008]. Alguns estudos [Santos et al. 2010, D'Abreu et al. 2011, Trentin et al. 2013] apontam o uso da RE como uma tecnologia aliada na resolução de problemas de diversas áreas do conhecimento.

Alguns kits robóticos apresentam suas próprias linguagens e ambientes de programação, que podem ser por meio de ícones ou de textos. Também é possível programá-los em linguagens de programação tradicionais como $\mathrm{C} / \mathrm{C}++$, Python e Java. Para realizar estudos desta natureza, faz-se necessário adquirir materiais e componentes que possuem um custo bastante elevado. [Trentin et al. 2013] sustentam essa afirmação, e ainda atestam que esses kits não possuem muita adaptabilidade no que diz respeito à interação com outros dispositivos que não pertençam ao fabricante ou escolhas de outras linguagens de programação não presentes no kit.

Existem várias formas de interagir com os robôs criados em projetos de robótica. Vários laboratórios remotos têm sido desenvolvidos, a exemplo de [Lima \& Siebra 2015; de Lima et al. 2016]. Além disso, alguns laboratórios remotos têm sido integrados a Ambientes Virtuais de Aprendizagem (AVAs), onde ele pode ser visto como um recurso adicional disponível para os usuários.

Este artigo tem como objetivo apresentar um Laboratório Remoto de Robótica (LRR) que disponibiliza equipamentos físicos para a realização de experimentos servindo como elemento motivador para a aprendizagem de conceitos iniciais de programação. A escolha da Robótica Educacional (RE) como objeto de experimentação científica atrelada a sistemas de ensino e aprendizagem tem-se demonstrado possuir uma vantagem significativa para os alunos através da melhoria do aprendizado. $\mathrm{O}$ laboratório desenvolvido foi integrado ao AVA Moodle, permitindo que os alunos tenham acesso aos materiais de ensino, além de possuir um espaço destinado para a prática de programação utilizando robôs.

Para relatar o trabalho desenvolvido, o restante do artigo está organizado da seguinte forma: a Seção 2 apresenta a metodologia da pesquisa, e a Seção 3 apresenta 
VI Congresso Brasileiro de Informática na Educação (CBIE 2017)

Anais do XXVIII Simpósio Brasileiro de Informática na Educação (SBIE 2017)

os trabalhos relacionados. A descrição do Laboratório Remoto de Robótica e a arquitetura do sistema são apresentadas na Seção 4. A Seção 5 apresentação a avaliação e resultados obtidos. Por fim a Seção 6 apresenta as conclusões e trabalhos futuros.

\section{Metodologia}

A metodologia desta pesquisa é de natureza qualitativa, pois o ambiente natural é a fonte direta para coleta de dados e não requer o uso de métodos e técnicas estatísticas [Gil 1991]. Do ponto de vista do seu objetivo é de natureza exploratória, pois envolve levantamento bibliográfico e entrevistas com pessoas que tiveram experiências práticas com o problema pesquisado [Gil 1991]. Para realização dos experimentos foi realizado um Estudo de Caso em uma escola pública, com alunos do $7^{\circ}$ e $8^{\circ}$ ano do Ensino Fundamental. Para avaliação da proposta, foi aplicado um questionário para que os alunos pudessem avaliar o laboratório desenvolvido. Foi necessário seguir uma série de procedimentos típicos de projetos desta natureza, tais como:

(1) Levantamento bibliográfico sobre os laboratórios remotos que permitem manipular e/ou programar robôs à distância;

(2) Desenvolvimento do sistema;

(3) Identificação dos critérios dos usuários que irão participar dos testes;

(4) Realização de testes com usuários reais. Esses testes envolveram usuários, com ou sem conhecimento de robótica a fim de testar e avaliar as funcionalidades do sistema;

(5) Aplicação de questionário com os usuários para avaliar se o sistema proposto motivou-os a aprender programação.

(6) Avaliação do sistema. Nessa fase foram analisados os resultados obtidos dos testes realizados com usuários reais. Os resultados serviram para validar a abordagem, e também foram fatores essenciais para melhoria do sistema.

\section{Trabalhos Relacionados}

As Instituições de Ensino Superior (IES) cujos cursos apresentam necessidades de experimentação laboratorial têm enfrentado muitas dificuldades quanto à qualidade de ensino. De acordo com [Victorino et al. 2009], a realização de trabalhos experimentais em laboratórios devidamente equipados é essencial para se aprender ciência e adquirir cultura científica. Porém, nem todos os jovens têm acesso a estes equipamentos, especialmente aqueles que estudam em instituições públicas. Esta seção apresenta alguns trabalhos desenvolvidos para apoiar o ensino de programação.

Um sistema que auxilia nas atividades de ensino/aprendizagem dos conceitos fundamentais da programação de computadores é apresentado no trabalho de [Martins et al. 2014]. O LogoBot implementa um subconjunto da linguagem de programação didática Logo, tradicionalmente utilizada no ensino de tais conceitos. Ainda voltado para estudantes iniciantes, [Carlos et al. 2016] apresentam o protótipo block.ino, que trata-se de uma interface adaptada para o desenvolvimento de programas computacionais para uma placa Arduino, controlando sensores e atuadores por meio de 
acesso remoto ao recurso. O protótipo é voltado para o ensino de lógica de programação, robótica e eletrônica básica.

Uma Revisão Sistemática da Literatura (RSL) feita por [Almeida \& Netto 2015] apresenta um estudo sobre laboratórios remotos que permitem programar robôs a distância. Os autores fazem uma análise das características, tecnologias e ferramentas utilizadas em cada um deles, além de apresentar as contribuições dos mesmos. Um ambiente de programação visual para RE é apresentado no trabalho de [Alves et al. 2015]. Este ambiente foi configurado para funcionar com o hardware Arduino, permitindo a usuários iniciantes programar um dispositivo robótico. Sua versão atual já é capaz de rodar na nuvem, bem como na máquina do usuário com qualquer sistema operacional.

O trabalho de [Lima \& Siebra 2015] apresenta uma plataforma aberta que está em fase de desenvolvimento, e possui um laboratório virtual para simular robôs, e um espaço remoto para programação de robôs reais. Não foi definida a linguagem de programação utilizada, e nem foram realizados testes. Neste mesmo sentido, o desenvolvimento de um laboratório remoto com duas interfaces de usuário para o ensino de programação e fundamentos da robótica usando placas Arduino é apresentado no trabalho de [de Lima et al. 2016]. A primeira interface usa linguagem de programação visual em um aplicativo móvel, para tornar a experiência mais atraente para aqueles que estão começando a programar. A segunda interface utiliza linguagem de programação textual, em uma página Web, semelhante a IDE original do Arduino, para aqueles usuários que pretendem usar uma linguagem de programação textual. Além disso, é apresentada uma avaliação de usabilidade do aplicativo usando a linguagem de programação visual para obter um feedback.

Todos os trabalhos apresentados nesta seção possuem um reconhecimento significativo junto ao meio acadêmico e serviram de motivação para o desenvolvimento desta pesquisa. Como diferencial, este trabalho possibilita que os usuários programem remotamente robôs Lego NXT, na linguagem de programação NXT-Python. Além disso, acredita-se que o desenvolvimento de um laboratório remoto de robótica, sirva como elemento motivador para a aprendizagem de programação, pois permitirá visualizar, explorar e manipular robôs reais, além de observar os efeitos destas alterações.

\section{Laboratório Remoto de Robótica}

O sistema desenvolvido nesta pesquisa encontra-se disponível em um AVA acessado via web, e pode ser acessado através do endereço <http://gaire.icomp.ufam.edu.br/ava> (apresentado na Figura 1). Neste ambiente, os alunos podem efetuar seu cadastro, conhecer novas pessoas, ter acesso aos materiais didáticos, agendar experimentos, realizar atividades e programar o robô remotamente. 
VI Congresso Brasileiro de Informática na Educação (CBIE 2017)

Anais do XXVIII Simpósio Brasileiro de Informática na Educação (SBIE 2017)



Figura 1. Página Inicial do Laboratório

O primeiro requisito para ter acesso à sala virtual é possuir um e-mail pessoal e se cadastrar no sistema. Em seguida, é enviado um e-mail de confirmação com instruções iniciais para acesso. Para ter acesso à sala de experimentação e iniciar a prática de programação, é sugerido aos alunos que efetuem uma reserva, observando os dias e horários disponíveis. Além disso, é necessário que eles leiam os materiais disponibilizados na sala virtual, que irão auxiliá-los na programação remota do robô. A Figura 2 apresenta a arquitetura do sistema.

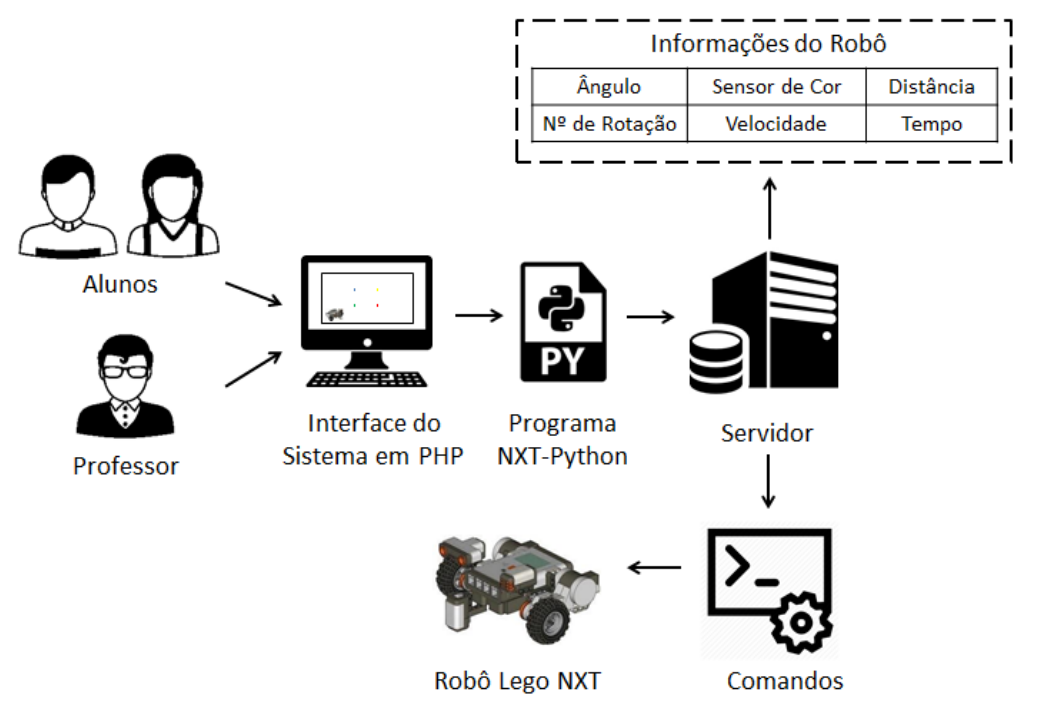

Figura 2. Arquitetura do Sistema

A Figura 2 apresenta também as ferramentas utilizadas no processo de comunicação entre os usuários e o kit robótico. Para tanto, utilizou-se 1 câmera IP para capturar as imagens do laboratório físico, o kit robótico Lego Mindstorms NXT e um conector Bluetooth USB 2.0 para enviar os comandos pro robô. Tanto professores como alunos podem ter acesso ao sistema através do AVA Moodle. O programa escrito na linguagem NXT-Python (Python para Lego NXT) é enviado pela interface do sistema, e as informações de todos os alunos são armazenadas no Banco de Dados (BD) do servidor $\mathrm{Web}$.

No laboratório há 5 atividades a serem realizadas, disponibilizadas sequencialmente por níveis de dificuldades, descritas no trabalho de [Almeida et al. 2017], sendo elas: (1) Descrição: Acender a luz verde do sensor de cor, e mantê-la acesa 
VI Congresso Brasileiro de Informática na Educação (CBIE 2017)

Anais do XXVIII Simpósio Brasileiro de Informática na Educação (SBIE 2017)

por 5 segundos. Pontuação: 10. Nível: Iniciante. (2) Descrição: Acender a luz vermelha do sensor de cor, mantê-la acesa por 5 segundos e desligá-la. Repetir o mesmo procedimento para a cor azul. Pontuação: 15. Nível: Básico. (3) Descrição: Acender a luz azul do sensor de cor, e mover o robô em linha reta por 10 segundos. Pontuação: 25. Nível: Intermediário. (4) Descrição: Acender a luz verde do sensor de cor, e mover o robô em linha reta até o final do tablado. Repetir o mesmo procedimento para a volta, porém desta vez com a luz vermelha acesa. Pontuação: 30. Nível: Avançado. (5) Descrição: Programar o robô para percorrer todo o tablado. Acender a luz azul do sensor de cor caso esteja andando em direção ao comprimento. Acender a luz verde do sensor de cor caso esteja andando em direção à largura. Pontuação: 40. Nível: Expert.

A atribuição do perfil de cada aluno está associada às atividades que já foram concluídas no laboratório, sendo atribuída uma pontuação automaticamente. A Figura 3 apresenta a sala de experimentação, onde os alunos submetem o código no espaço destinado para tal, e caso não contenha erros sintáticos ele visualiza na imagem da câmera o robô executando as ações conforme programado. A análise sintática do código é realizada pelo sistema tão logo um código tenha sido enviado, e um feedback é fornecido ao aluno.

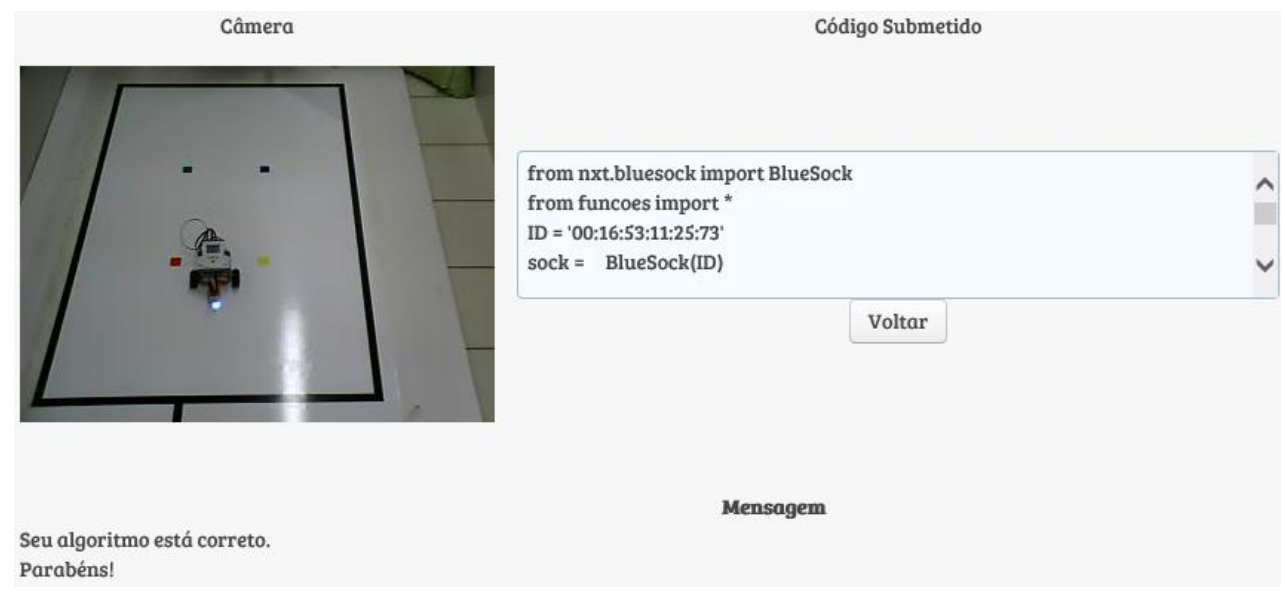

Figura 3. Sala de Experimentação

O sistema possibilita aos alunos a realização de experimentos com o kit robótico Lego Mindstorms NXT. Neste sentido, é permitido que os alunos programem os robôs com a linguagem de programação NXT-Python, e não apenas manipulem o por meio de setas de controle. Os trabalhos apresentados na Seção 2 colaboraram e motivaram seu desenvolvimento. Os testes realizados e os resultados obtidos são apresentados na próxima seção.

\section{Avaliação e Resultados Obtidos}

Os testes tiveram a duração de 2 semanas e foram realizados com alunos do $7^{\circ}$ e $8^{\circ}$ ano do Ensino Fundamental do Colégio de Aplicação da Universidade Federal de Roraima (CAP/UFRR). Para participação na atividade, os alunos receberam da Coordenadora Pedagógica da escola um Termo de Consentimento Livre e Esclarecido (TCLE) para que pudessem solicitar a anuência dos seus responsáveis. Após entregarem as autorizações devidamente assinadas, foi realizado o primeiro encontro (Figura 4) com o 
VI Congresso Brasileiro de Informática na Educação (CBIE 2017)

Anais do XXVIII Simpósio Brasileiro de Informática na Educação (SBIE 2017)

objetivo de explanar o funcionamento do laboratório, atividades e funções disponíveis para programar o robô remotamente.



Figura 4. Primeiro Contato com os Alunos

Ainda nesse primeiro contato, foi solicitado que os alunos se cadastrassem no sistema e agendassem o experimento no horário oposto das aulas. Com o objetivo de caracterizar os alunos participantes dos experimentos, foi aplicado um questionário para saber o perfil deles e o conhecimento prévio que tinham em programação. No total, 14 alunos entre 12 e 16 anos, participaram dos experimentos. Todos afirmaram estar familiarizados com a utilização do computador, e mesmo os que não possuem computador pessoal utilizam o disponibilizado na escola.

Como neste trabalho o laboratório remoto foi integrado a um AVA, foi necessário saber o conhecimento dos alunos em relação à navegabilidade na Internet. Neste sentido, 62\% afirmaram que estavam acostumados a navegar e sabiam navegar bem, $29 \%$ afirmaram saber navegar bem e somente $9 \%$ afirmou saber navegar moderadamente. Com relação aos conhecimentos prévios em programação, 29\% afirmaram não ter qualquer conhecimento, $33 \%$ afirmaram ter pouco conhecimento, $24 \%$ afirmaram ter algum conhecimento e apenas $14 \%$ afirmaram ter muito conhecimento. Os gráficos representando essas duas questões são apresentados abaixo na Figura 5.



Figura 5. Conhecimentos em navegar na internet e em programação

De acordo com [Printrich \& Schunk 2002], a motivação para a aprendizagem pode ser avaliada por meio de observações diretas de comportamentos, pelo julgamento de outros e por relatos e autoavaliações. As observações diretas estão relacionadas à 
VI Congresso Brasileiro de Informática na Educação (CBIE 2017)

Anais do XXVIII Simpósio Brasileiro de Informática na Educação (SBIE 2017)

análise dos comportamentos de um estudante que poderiam ser indicativos de aspectos motivacionais. Sendo assim, ao término dos experimentos, uma pesquisa de opinião foi disponibilizada na sala virtual, com o objetivo de analisar a impressão dos alunos após terem experiências com robótica no laboratório remoto.

Todos os alunos avaliaram a metodologia utilizada como muito importante e concordaram que a utilização do robô associado à aprendizagem de programação proporcionou uma experiência positiva. Além disso, 96\% dos alunos demonstraram-se motivados e interessados em continuar utilizando o laboratório remoto de robótica para aprender mais conceitos relacionados à programação, e questionaram se novas atividades seriam disponibilizadas. A Figura 6 apresenta os gráficos correspondentes a essas questões.

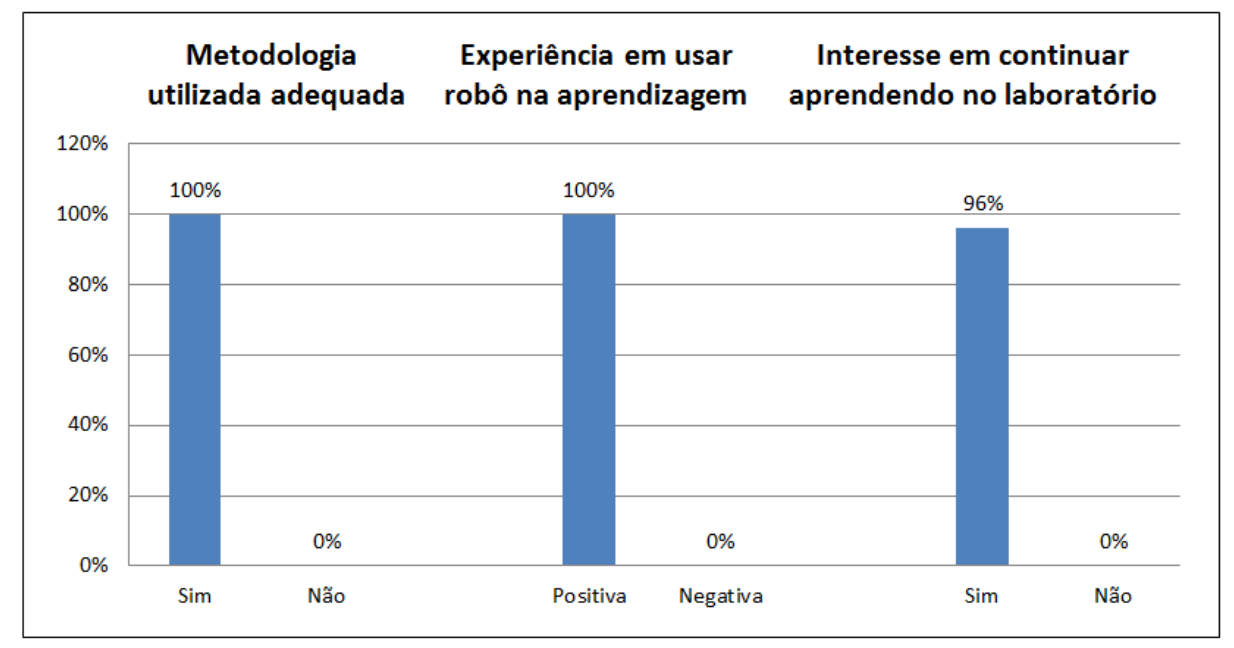

Figura 6. Conhecimentos em navegar na internet e em programação

Foi possível observar que a cada atividade que os alunos realizavam com êxito, aguçava a curiosidade para saber quais seriam as próximas. No mesmo sentido, eles calculavam a pontuação obtida nos experimentos, e ajudavam os colegas que estavam com algum tipo de dificuldade. Com base nesses resultados, podemos concluir que o trabalho apresentado neste artigo colaborou para aumentar o interesse dos alunos nessa área, motivando-os na busca de mais conhecimentos relacionados à programação e robótica. Como limitação, para o correto funcionamento do sistema, é necessário que a sala de desafios seja acessada pelo navegador Internet Explorer, para a correta exibição da imagem da câmera que utiliza o plugin ActiveX.

\section{Conclusões e Trabalhos Futuros}

Este artigo apresentou uma abordagem de ensino de programação que serviu como elemento motivador para a aprendizagem utilizando robôs, proporcionando aos alunos a oportunidade de ter contato com experimentos desta natureza. Nosso propósito por intermédio dessa abordagem foi investigar se os alunos se sentiriam motivados a aprenderem programação e robótica. A motivação para aprender é considerada essencial nos sistemas educacionais.

Foi necessário familiarizar os alunos com as tecnologias voltadas a computadores e robôs, dar suporte sobre o funcionamento do sistema, explanar os 
VI Congresso Brasileiro de Informática na Educação (CBIE 2017)

Anais do XXVIII Simpósio Brasileiro de Informática na Educação (SBIE 2017)

conteúdos e a metodologia a ser utilizada. Além disso, foram realizados diversos testes para detectar a presença de falhas no sistema. A análise dos registros armazenados no $\mathrm{BD}$ e o feedback dos alunos envolvidos na pesquisa, colaboraram para que essas falhas fossem corrigidas.

Os resultados evidenciam que o sistema é viável de ser utilizado por qualquer pessoa que tenha interesse em aprender programação e robótica, possua conhecimentos básicos em informática e saiba navegar na Web. A abordagem apresenta avanços sobre os laboratórios remotos de robótica encontrados na literatura, pois permite selecionar desafios distintos, programando os robôs em uma linguagem de programação. Vale ressaltar que essa proposta não pretende substituir os laboratórios físicos existentes, mas sim expandir ao maior número de pessoas a oportunidade de ter acesso remoto aos robôs reais.

O sistema desenvolvido está funcional, e com pequenas adaptações pode ser extensível para aceitar outras linguagens de programação. Da mesma maneira, é possível modificá-lo e disponibilizá-lo em uma página $W e b$, tornando-o independente da estrutura do AVA Moodle. Como trabalhos futuros pretende-se substituir os kits robóticos Lego Mindstorms pelo Arduino, devido a este possuir menor custo e ser mais flexível para aceitar outras linguagens de programação. Pretende-se ainda desenvolver um ambiente, onde seja possível analisar o histórico dos alunos, identificando dificuldades individuais de aprendizagem, e sugerindo atividades específicas para cada um.

\section{Referências}

Almeida, T. O., \& Netto, J. F. (2015). "Robótica Pedagógica Aplicada ao Ensino de Programação: Uma Revisão Sistemática da Literatura”. In XXVI Simpósio Brasileiro de Informática na Educação. (Vol. 26, No. 1, p. 597).

Almeida, T. O., Netto, J. F., \& Rios, M. L. (2017). "Remote Robotics Laboratory as Support to Teaching Programming". In 47th Annual Frontiers in Education (FIE) Conference.

Alves, R. M., Sampaio, F. F., \& da Fonseca, E. M. (2015). "Duinoblocks: Desenho e implementação de um ambiente de programação visual para robótica educacional". Revista Brasileira de Informática na Educação, 22(03):126.

Azevedo, S.; Aglaé, A.; Pitta, R. (2010). "Minicurso: Introdução à Robótica Educacional". $62^{\mathrm{a}}$ Reunião Anual da SBPC. Disponível em:< http://www.sbpcnet.org.br/livro/62ra/minicursos/MC\%20Samuel\%20Azevedo.pdf $>$. Acessado dia 14/04/2017.

Carlos, L. M., de Lima, J. P., Simão, J. P. S., \& Silva, J. (2016). "block.ino: Um experimento remoto para ensino de lógica de programação, robótica e eletrônica básica". In Anais dos Workshops do $\mathrm{V}$ Congresso Brasileiro de Informática na Educação (Vol. 5, No. 1, p. 151).

CSTA. (2013). "CSTA National Secondary Computer Science Survey: Comparison of Results from 2005, 2007, 2009, 2011 and 2013”. Disponível em: $<$ http:/csta.hosting.acm.org/csta/csta/Research/sub/Projects/ResearchFiles/CSTASur vey2013Comp.pdf $>$. Acessado dia 27/06/2017. 
VI Congresso Brasileiro de Informática na Educação (CBIE 2017)

Anais do XXVIII Simpósio Brasileiro de Informática na Educação (SBIE 2017)

D'Abreu, J. V. V., Mirisola, L. G., \& Ramos, J. J. (2011). “Ambiente de robótica pedagógica com br_gogo e computadores de baixo custo: Uma contribuição para o ensino médio". In Anais do XXII Simpósio Brasileiro de Informática na Educação.

de Lima, J. P. C., Carlos, L. M., Simão, J. P. S., Pereira, J., Mafra, P. M., \& da Silva, J. B. (2016). "Design and implementation of a remote lab for teaching programming and robotics". IFAC-PapersOnLine, 49(30), 86-91.

Gil, A. C. (1991). "Como elaborar projetos de pesquisa”. São Paulo: Atlas.

Gomes, A., Areias, C., Henriques, J., \& Mendes, A. J. (2008). “Aprendizagem de programação de computadores: dificuldades e ferramentas de suporte". Revista Portuguesa de Pedagogia, (42-2):161-179.

Lima, E., \& Siebra, C. (2015). "Especificação de uma Plataforma Aberta para o Ensino de Robótica a Distância”. In Anais do XXVI Simpósio Brasileiro de Informática na Educação. (Vol. 26, No. 1, p. 414).

Martins, J. P. d. A., Rocha, C. A., Ramos, B. A., \& Vanderlei, I. M. (2014). "Logobotum sistema robótico simulador da linguagem logo para auxílio no aprendizado de programação". In Anais do XXV Simpósio Brasileiro de Informática na Educação.

Printrich, P. R., \& Schunk, D. H. (2002). "Motivation in education: theory, research, and application". Columbus, OH: Merrill Prentice Hall.

Santos, A., Gomes, A., \& Mendes, A. (2013). "A taxonomy of exercises to support individual learning paths in initial programming learning". In Frontiers in Education Conference, 2013 IEEE, pages 87-93. IEEE.

Santos, F. L., Nascimento, F. M. S., \& Bezerra, R. M. (2010). "Reduc: A robótica educacional como abordagem de baixo custo para o ensino de computação em cursos técnicos e tecnológicos". In Anais do Workshop de Informática na Escola (Vol. 1, No. 1, pp. 1304-1313).

Tavares, O. L., de Menezes, C. S., \& de Nevado, R. A. (2012). "Pedagogical architectures to support the process of teaching and learning of computer programming". In Frontiers in Education Conference (FIE), (pp. 1-6). IEEE.

Trentin, M. A., Pérez, C. A. S., \& Teixeira, A. C. (2013). “A robótica livre no auxílio da aprendizagem do movimento retilíneo". In Anais do XIX Workshop de Informática na Escola, volume 1, pages 51-59.

Victorino, L., Elia, M., Gomes, Â., de Castro, M., \& Bastos, C. (2009). "Laboratório Virtual de Atividades Didáticas - LabVad”. In Anais do Workshop de Informática na Escola (Vol. 1, No. 1, pp. 1723-1732). 